\title{
PATZ1 interacts with p53 and regulates expression of p53-target genes enhancing apoptosis or cell survival based on the cellular context
}

\author{
T Valentino ${ }^{1,2,4}$, D Palmieri ${ }^{3,4}, M_{\text {Vitiello }}^{1,2}$, GM Pierantoni ${ }^{1,2}$, A Fusco $^{1,2}$ and M Fedele ${ }^{\star, 1,2}$
}

PATZ1 is a transcriptional factor functioning either as an activator or a repressor of gene transcription depending upon the cellular context. It appears to have a dual oncogenic/anti-oncogenic activity. Indeed, it is overexpressed in colon carcinomas, and its silencing inhibits colon cancer cell proliferation or increases sensitivity to apoptotic stimuli of glioma cells, suggesting an oncogenic role. Conversely, the development of B-cell lymphomas, sarcomas, hepatocellular carcinomas and lung adenomas in Patz1-knockout (ko) mice supports its tumour suppressor function. PATZ1 role in mouse lymphomagenesis is mainly because of the involvement of PATZ1 in BCL6-negative autoregulation. However, this does not exclude that PATZ1 may be involved in tumorigenesis by other mechanisms. Here, we report that PATZ1 interacts with the tumour suppressor p53 and binds p53-dependent gene promoters, including those of BAX, CDKN1A and MDM2. Knockdown of PATZ1 in HEK293 cells reduces promoter activity of these genes and inhibits their expression, suggesting a role of PATZ in enhancing p53 transcriptional activity. Consistently, Patz1-ko mouse embryonic fibroblasts (MEFs) show decreased expression of Bax, Cdkn1a and Mdm2 compared with wild-type (wt) MEFs. Moreover, Patz1-ko MEFs show a decreased percentage of apoptotic cells, either spontaneous or induced by treatment with 5 -fluorouracil $(5 \mathrm{FU})$, compared with wt controls, suggesting a pro-apoptotic role for PATZ1 in these cells. However, PATZ1 binds p53-target genes also independently from p53, exerting, in the absence of p53, an opposite function on their expression. Indeed, knockdown of PATZ1 in p53-null osteosarcoma cells upregulates BAX expression and decreases survival of 5FU-treated cells, then suggesting an anti-apoptotic role of PATZ1 in p53-null cancer cells. Therefore, these data support a PATZ1 tumour-suppressive function based on its ability to enhance p53-dependent transcription and apoptosis. Conversely, its opposite and anti-apoptotic role in p53-null cancer cells provides the perspective of PATZ1 silencing as a possible adjuvant in the treatment of p53-null cancer.

Cell Death and Disease (2013) 4, e963; doi:10.1038/cddis.2013.500; published online 12 December 2013

Subject Category: Cancer

The human PATZ1 gene, also known as MAZR, ZSG or ZNF278, encodes four alternatively expressed proteins, ranging from 537 to 687 amino acids, that share a common modular structure consisting of a POZ domain, an AT hook and four to seven $\mathrm{C} 2 \mathrm{H} 2$ zinc fingers. ${ }^{1-3}$ According to these domains, PATZ1 is a member of the POK (POZ and kruppellike zinc finger) family, an unique group of transcription factors having key roles in development and cancer through their involvement in a variety of cellular processes, including cell proliferation, senescence and apoptosis. ${ }^{4,5}$ Many POK proteins, such as $\mathrm{HIC}-1$, Bcl6, PLZF, Nac-1 and others, have been linked directly or indirectly to $p 53$ regulation, ${ }^{5-7}$ and PATZ1 itself has been recently shown to inhibit endothelial cell senescence through a p53-dependent pathway. ${ }^{8}$
However, the mechanism of action of these proteins is largely unknown.

As for other POK family members, the transcriptional activity of PATZ is dependent on the POZ-mediated oligomer formation, suggesting PATZ1 as an architectural transcription factor rather than a typical transactivator, thus working either as activator or repressor depending on the presence of the interacting proteins in the cellular context. Consistently, PATZ1 has been reported to either activate or repress $c$-myc, ${ }^{1,2}$ to activate mast cell protease 6 and $F G F 4,{ }^{2,9}$ and to repress androgen receptor, $C D 8$ and $B C L 6$ genes. ${ }^{10-13}$

Several studies indicate a role of PATZ1 in carcinogenesis; however, it has not defined yet whether it behaves as a tumour suppressor or an oncogene. In fact, the PATZ1 gene has been

\footnotetext{
${ }^{1}$ Istituto di Endocrinologia e Oncologia Sperimentale (IEOS), CNR, Naples, Italy; ${ }^{2}$ Dipartimento di Medicina Molecolare e Biotecnologie Mediche, Università di Napoli "Federico II", Naples, Italy and ${ }^{3}$ Department of Molecular Virology, Immunology and Medical Genetics, Comprehensive Cancer Center, The Ohio State University, Columbus, OH, USA

*Corresponding author: M Fedele, Istituto di Endocrinologia e Oncologia Sperimentale (IEOS), National Research Council, Via S. Pansini, 5-80127 Napoli, Italy. Tel: +39 81 7463054; Fax: +39 81 7463749. E-mail: mfedele@unina.it

${ }^{4}$ These authors contributed equally to this work.

Keywords: apoptosis; cell survival; tumour suppressor; oncogene; gene regulation

Abbreviations: Ko, knockout; MEFs, mouse embryonic fibroblasts; 5FU, 5-fluorouracil; POK, POZ and kruppel-like zinc finger; HA, hemagglutinin; Ab, antibody; GST, glutathione S-transferase; SDS, sodium dodecyl sulphate; SDS-PAGE, polyacrylamide-SDS gel electrophoresis; IP, immunoprecipitation; ChIP, chromatin immunoprecipitation; sh, short hairpins; CTRL, control; wt, wild-type; DLBCLs, diffuse large B-cell lymphomas; S.E., standard error; S.D., standard deviation; PCR, polymerase chain reaction; RT-PCR, reverse transcription PCR; qPCR or qRT-PCR, quantitative PCR or RT-PCR

Received 16.7.13; revised 06.11.13; accepted 13.11.13; Edited by G Melino
} 
found to be rearranged with the EWS gene in a small round cell sarcoma where the other PATZ1 allele is lost. ${ }^{3}$ Moreover, loss of heterozygosity has been found at the FRA22B fragile site, where the PATZ1 gene is located, in several solid tumours, ${ }^{14}$ then supporting a potential tumour suppressor role for PATZ1. Furthermore, both heterozygous and homozygous Patz1-knockout (ko) mice spontaneously develop several tumours, including BCL6-expressing Non-Hodgkin lymphomas, sarcomas and hepatocellular carcinomas, ${ }^{13}$ and Patz1null mouse embryonic fibroblasts (MEFs) showed increased expression of various proteins involved in cell cycle activation, including cyclin D2, CDK4, Cyclin E, HMGA1 and HMGA2, even though they also express abundant levels of cell cycle inhibitors, arrest in both G0/G1 and G2/M phases of the cell cycle and undergo premature senescence. ${ }^{15}$

On the other hand, PATZ1 overexpression has been described in various human malignant neoplasias, including colon, testicular and breast tumours, ${ }^{16-18}$ and PATZ1 downregulation by siRNA either blocks the growth of colorectal carcinoma cells ${ }^{16}$ or increases sensitivity of glioma cell lines to apoptotic stimuli. ${ }^{19}$

We have previously demonstrated that a critical mechanism for the development of B-cell lymphoma in Patz1-ko mice relies on the increased $\mathrm{Bcl} 6$ expression levels consequent to the lack of negative regulation by PATZ1. ${ }^{13}$ However, other mechanisms may be envisaged, especially those involved in the development of solid tumours, such as hepatocarcinomas and lung adenomas in Patz1-ko mice.

In order to elucidate other possible mechanisms by which PATZ1 may be involved in carcinogenesis, we decided to search for PATZ1-interacting proteins. To this aim, we screened an antibody $(A b)$ array that allowed us to identify several potential PATZ1 interactors. Then, we focused on the p53 tumour suppressor because of its widely demonstrated role in cancer. ${ }^{20}$

We first validated the PATZ1/p53 interaction by co-immunoprecipitating the endogenous proteins in mammalian cells, and then we demonstrated that the PATZ1/p53 complex is present on p53-targeted genes, where PATZ1 enhances p53 transcriptional activity. Next, we showed that PATZ binds p53-targeted genes in p53-null Saos-2 cells, where it regulates transcription in an opposite manner compared with p53. Finally, we showed that PATZ1 is endowed of both pro-apoptotic and anti-apoptotic activities, depending on the cellular context.

\section{Results}

PATZ is in the same complex with p53. In order to identify new PATZ1-interacting proteins, we employed an Ab array containing hundreds of high-quality antibodies against well-studied proteins, involved in cell cycle regulation, apoptosis and signal transduction. The array was incubated with total cell extracts from HEK293 cells transfected with the human full-length PATZ1 cDNA, tagged with the HA-epitope and immunoblotted with anti-HA $A b$. The results indicated an interaction between PATZ1 and several proteins (Supplementary Figure S1a); among these, we focused our attention on the oncosuppressor p53 because of its relevance in cancer pathogenesis.

To confirm the interaction between PATZ1 and p53, endogenous PATZ1 and p53 were co-immunoprecipitated in total cell extracts from HEK293 with anti-p53 Ab and analysed by western blotting with anti-PATZ1 Ab (Figure 1a, upper panel). Western blot with monoclonal p53 Ab confirmed the correct immunoprecipitation (IP) of the p53 protein (Figure 1a, lower panel). The reciprocal experiment performed immunoprecipitating with anti-PATZ1 Ab and revealing with anti-p53 $\mathrm{Ab}$ confirmed the interaction (Supplementary Figure S1b). Consistent with the specificity of this interaction, no co-IP was observed when nonspecific IgG was used to immunoprecipitate. These results demonstrate that PATZ1 and p53 are found in the same complexes in mammalian cells.

To identify the p53 domain involved in the interaction with PATZ1, pull-down assays were performed using HEK293 total cell extracts and two bacterially expressed p53 deletion mutants fused to GST (Figure 1b). ${ }^{21}$ HA-PATZ1 was transiently transfected into HEK293 cells that were harvested $48 \mathrm{~h}$ later. Protein extracts were tested for their interaction with the GST-p53 deletion mutants. The complexes were immobilized on a glutathione-Sepharose matrix, separated by polyacrylamide-SDS gel electrophoresis (SDS-PAGE) and a

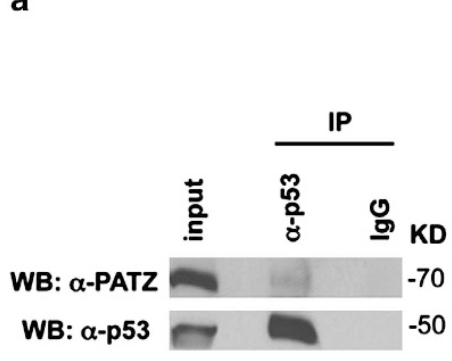

b

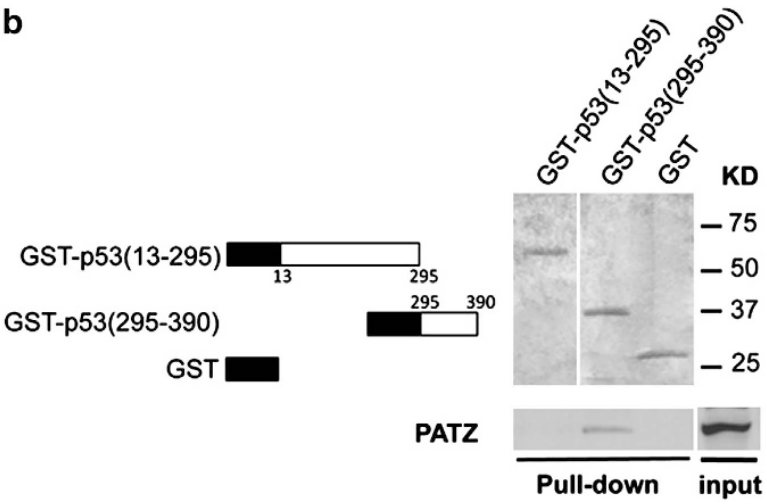

Figure 1 PATZ and p53 are in the same complex. (a) Co-immunoprecipitation of endogenous PATZ and p53 in HEK293 cells. (b) GST pull-down assay of the GST-p53 fusion proteins represented on the left with cell lysates of HEK293 cells. The bound complexes were separated by SDS-PAGE and the filter was incubated with anti-PATZ Ab (lower panel). A parallel twin gel was stained with coomassie-blue to show equal amount of the GST-fusion proteins used in the assay (upper panel). IgG $=$ nonspecific antibody; input = total cell lysates 
blotted, and the filters were hybridized with an anti-HA Ab. The GST-p53(295-390) mutant keeps the ability to bind PATZ1, whereas no binding was observed with the GST-p53(13-295) (Figure 1b, lower panel). No PATZ1 was detectable in the complexes obtained with the GST protein alone (Figure $1 \mathrm{~b}$, lower panel). Equal amounts of GST-fusion proteins were loaded in the pull-down assay, as shown by the blueCoomassie staining of a parallel twin gel (Figure 1b, upper panel). These results suggest that the $\mathrm{C}$-terminal domain of p53, which comprises the tetramerization (aa 323-356) and the regulatory (363-393) domains, is responsible for the interaction with PATZ1.

PATZ1 binds BAX, CDKN1A and MDM2 promoters together or not with p53. p53 is a sequence-specific transcription factor whose DNA-binding consensus is present in a large number of promoters. $^{22}$ Similarly, PATZ1 is a transcription factor that binds DNA to specific consensus sequences in promoter regions of several genes. ${ }^{23}$ Thus, we asked whether the physical interaction between PATZ1 and p53 takes place on promoter regions of known p53 targets. Therefore, we evaluated whether PATZ1 protein binds the promoters of p53-target genes, such as $B A X, M D M 2$ and $C D K N 1 A$, by performing chromatin IP (ChIP) assays. Chromatin from Raji cells was crosslinked and immunoprecipitated with anti-PATZ1 or nonspecific IgG. Immunoprecipitated chromatin was then analysed by $\mathrm{PCR}$, using primers spanning the $-250 /-530$ region of $B A X$, the $-400 /-100$ region of MDM2 and the $-1550 /-1200$ region of $C D K N 1 A$ previously shown to co-immunoprecipitate with $\mathrm{p} 53 .{ }^{21}$ Occupancy by PATZ1 of the above indicated promoter regions was detectable in anti-PATZ1-precipitated chromatin. Conversely, no precipitation was observed with IgG precipitates, and when primers for the control promoter $L P L$ were used, indicating that the binding of PATZ1 is specific for the selected promoters (Figure 2a). To determine whether PATZ1 occupies these promoter regions along with p53, we performed ChIP and Re-ChIP analysis on HEK293 cells transiently transfected with HA-tagged-PATZ1, p53 or both expression vectors. Cells were crosslinked and immunoprecipitated (ChIP) with anti-HA Ab, and then re-immunoprecipitated (Re-ChIP) with anti-p53 Ab. In particular, a part of the anti-HA-immunoprecipitated chromatin was analysed using quantitative $\mathrm{PCR}$ for $\mathrm{BAX}, \mathrm{MDM} 2$ and $C D K N 1 A$ promoter amplification, confirming the binding of PATZ to these promoters (Figure 2b). Another part of the PATZ1 complexes was subjected to Re-ChIP with anti-p53 $\mathrm{Ab}$, and then analysed using real-time PCR for BAX, MDM2 and CDKN1A promoters. The results shown in Figure $2 \mathrm{c}$ demonstrate that PATZ1 and p53 take part to the same complex on BAX and $C D K N 1 A$ but not on MDM2 promoters. The reciprocal experiments, using anti-p53 Ab for the first ChIP and antiHA antibodies for the Re-ChIP, confirmed the results (Supplementary Figure S2). It is worth noting that ChIP and Re-ChIP experiments have been performed on exogenously expressed proteins because of the difficulty to obtain good results when endogenous proteins are not abundantly expressed.

Taken together, these results indicate that PATZ1 binds the human $B A X, M D M 2$ and $C D K N 1 A$ promoters in vivo, and participate to the same DNA-bound complexes that contain p53 on the BAX and CDKN1A genes.

Interestingly, the PATZ1 protein was also capable of binding $B A X, M D M 2$ and $C D K N 1 A$ promoters in the osteosarcoma-derived p53-null Saos-2 cells (Figure 2d), suggesting that $p 53$ is not required for the binding of PATZ1 to these a
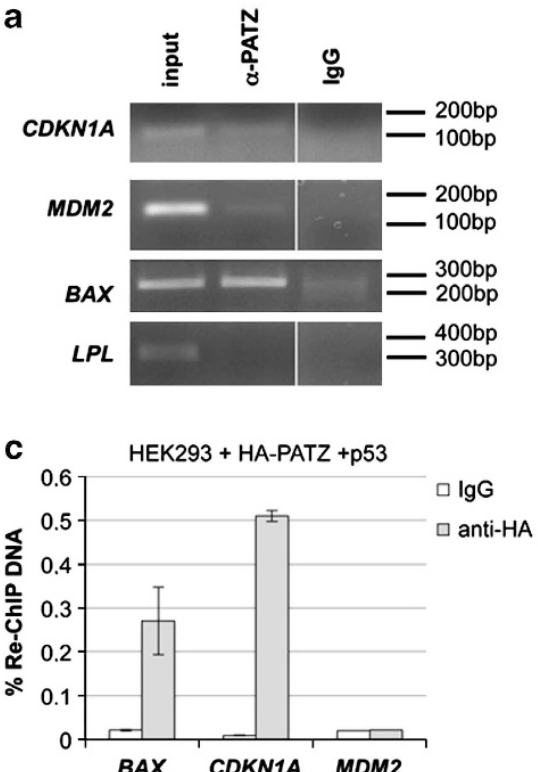

b
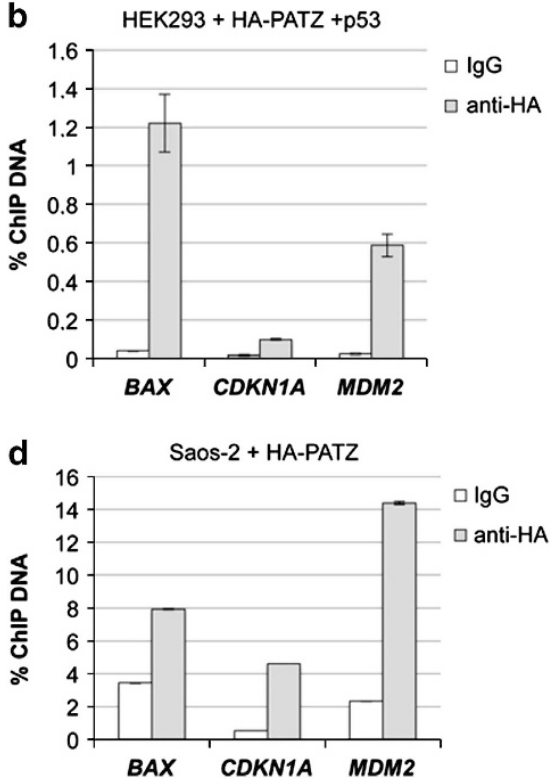

Figure 2 Binding of PATZ to p53-target genes. (a) ChIP assay in Raji cells to detect the endogenous in vivo binding of PATZ to CDKN1A, MDM2 and BAX gene promoters, as indicated. LPL gene promoter was also analysed as a negative control. (b) ChIP assay, revealed by real-time PCR, in HEK293 cells, transiently transfected with HA-PATZ and p53, to detect the binding of PATZ to the indicated gene promoters. (c) Re-ChIP assay of the samples shown in $\mathbf{b}$ to detect the binding of p53 to PATZ/DNA complexes on the indicated gene promoters. (d) ChIP assay in Saos-2 cells, transiently transfected with HA-PATZ, to detect the binding of PATZ to the indicated gene promoters. Mean values \pm S.D. of triplicates are shown in $\mathbf{b}-\mathbf{d}$ ). $\lg G=$ nonspecific $A b$ 
promoters. Moreover, by in silico analysis, using the TraFac Homology Server, ${ }^{24}$ we found the specific responsive elements of PATZ1 (here indicated as MAZR) in the promoter regions of $M D M 2$ and $C D K N 1 A$, suggesting that the binding of PATZ1 to these genes may be direct (Supplementary Table S1).

PATZ1 regulates transcription of p53-target genes. To study the functional consequences of the complex including PATZ1 and p53 on p53-target genes, we used reporter constructs driving luciferase gene expression under control of the BAX (BAX-luc), MDM2 (MDM2-luc) and CDKN1A (p21-luc) promoters. HEK293 cells were co-transfected with each of these reporter constructs together with plasmids expressing PATZ1, p53 or both proteins. As expected, p53 expression resulted in the upregulation of these promoters. No significant differences were observed when PATZ1 was transfected alone, whereas co-transfection of PATZ1 and p53 significantly enhances BAX promoter activity compared with that obtained by p53 alone, but had no significant effect on the other two promoters (Supplementary Figure S3). As HEK293 cells express abundant endogenous levels of PATZ1 (Figure 3a), we decided to analyse the p53-responsive promoter activities in HEK293 cells interfered for PATZ1. The knockdown of PATZ1 in these cells, by stable transfection of specific short hairpin (sh)-RNA (ShPATZ1) (Figure $3 \mathrm{~b}$ ), resulted in the reduction of $B A X$, MDM2 and CDKN1A promoter activities and/or gene expression with respect to the control cells (ShCTRL) (Figures $3 c$ and d). Furthermore, differently from ShCTRL + p53-transfected cells, in which activities of all the promoters analysed were significantly upregulated compared with ShCTRL controls, in ShPATZ1 + p53-transfected cells CDKN1A and MDM2 promoter activities were not significantly upregulated compared with ShPATZ1 controls, suggesting that $\mathrm{p} 53$ is not able to transactivate CDKN1A and MDM2 promoters in ShPATZ1 HEK293 cells (Figure 3c). Consistently, Patz1 ${ }^{+/}$MEFs showed decreased levels of endogenous Bax, Mdm2 and Cdkn1a mRNA, compared with control wild-type (wt) cells (Figure $3 e$ ). These results suggest that PATZ1 activate expression of the MDM2, CDKN1A and $B A X$ genes in both HEK293 cells and MEFs, and that it is required for proper $\mathrm{p} 53$ activity on MDM2 and CDKN1A promoters.

As we showed that PATZ1 is able to bind p53-dependent gene promoters also in the absence of p53, we also analysed the affects of PATZ1 knockdown on their transcriptional
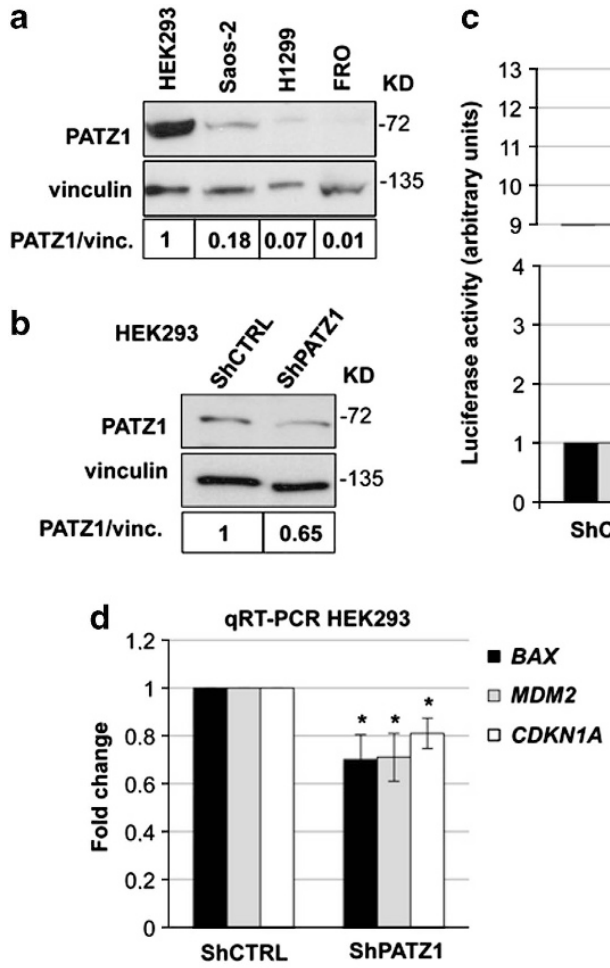
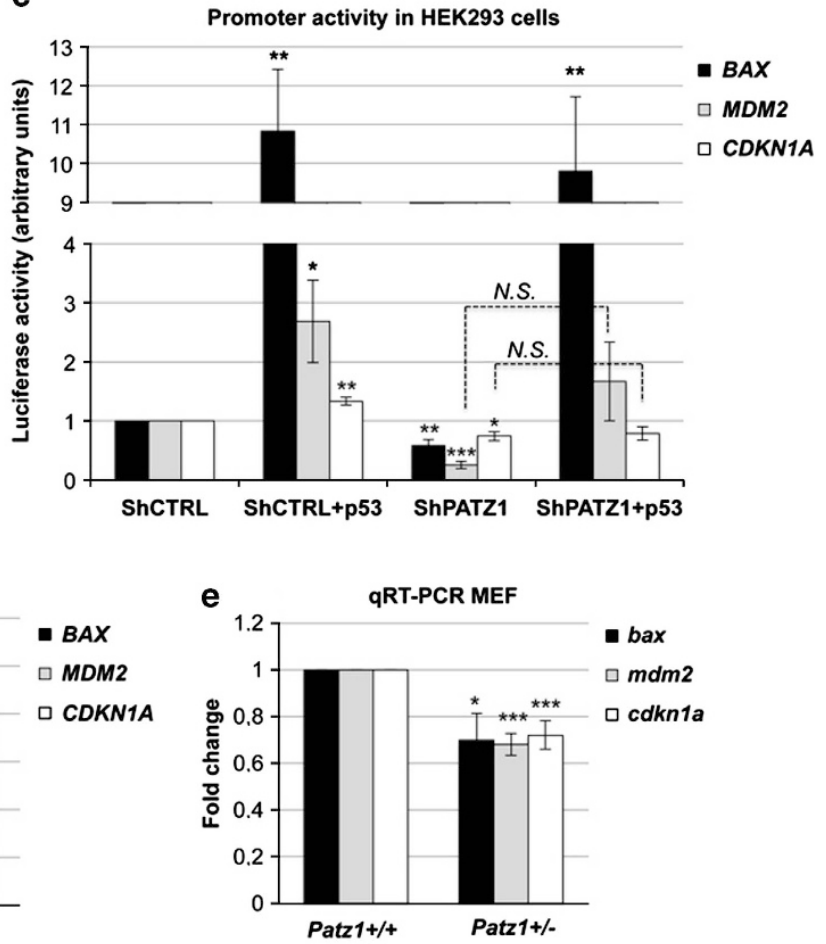

Figure 3 PATZ1 knockdown and knockout downregulates p53 activity and p53-target genes in HEK293 cells and MEFs. (a) Western blot analysis of PATZ expression in different cell lines, as indicated above the panels. Vinculin expression was evaluated as a loading control. Relative expression levels, compared with HEK293 cells and normalized with respect to vinculin, are indicated on the bottom. (b) Western blot analysis of PATZ expression in Short hairpin (Sh)PATZ1 (ShPATZ1) and backbone vector (ShCTRL)-expressing HEK293 cells. Vinculin expression was evaluated as a loading control. Relative expression levels, compared with ShCTRL cells and normalized with respect to vinculin, are indicated on the bottom. (c) Promoter activity of the BAX,CDKN1A and MDM2 genes as assessed by luciferase assay in cells shown in $\mathbf{b}$. Where indicated, p53 was co-transfected with the reporter plasmids. The data shown express the relative mean values \pm S.E., compared with ShCTRL, of three or four independent experiments, each one performed in duplicate. Asterisks indicate the statistical significance compared with ShCTRL. N.S. = not significant. (d) qRT-PCR analysis of BAX, MDM2 and CDKN1A gene expression in ShCTRL and ShPATZ1 cells. Expression levels are normalized to GAPDH, and the levels in ShCTRL cells were set as 1. Mean values \pm S.E. of at least four independent experiments are shown. Asterisks indicate the statistical significance versus control cells. (e) qRT-PCR analysis of bax, mdm2 and cdkn1a gene expression (normalized to Gapdh) in MEFs from Patz $1^{+/+}$and Patz $1^{+/-}$mice. Expression levels in Patz $1^{+1+}$ samples were set to 1 . The data shown express the mean values \pm S.E. of at least four experiments. Asterisks indicate the statistical significance compared with Patz $1^{+/+}$MEFs 
regulation in p53-null cells. To this aim, Saos-2 cells, interfered for the PATZ1 gene, were transfected with the reporter vectors above indicated, with or without a plasmid expressing p53, and luciferase activity was analysed (Figures $4 \mathrm{a}$ and $\mathrm{b}$ ). As shown in Figure $4 \mathrm{a}$, the activities of $B A X, M D M 2$ and $C D K N 1 A$ promoters were increased in cells interfered for PATZ1 (ShPATZ1) compared with control cells (ShCTRL). Moreover, the activity of a co-transfected p53 had a trend to be enhanced in ShPATZ1 cells compared with their control. Consistently, the endogenous levels of $B A X, M D M 2$ and CDKN1A mRNA in Saos-2 cells interfered for PATZ1 were upregulated compared with control cells (Figure 4c). To verify whether the different behaviour of PATZ in Saos-2 cells compared with HEK293 cells depends on the absence of p53, H1299 cells - another p53-null cancer cell line - were used to analyse the effect of the expression of PATZ on the BAX promoter, representing one of the above reported promoters. The results shown in Supplementary Figure S4 confirm also in this cell line an inhibitory effect of PATZ1 expression on $B A X$ activity.

Therefore, at odds with the data obtained in HEK293 cells and MEFs, which endogenously express p53, these results indicate that PATZ1 downregulates the expression of the $B A X, M D M 2$ and $C D K N 1 A$ genes in p53-null cells and suggest an oncogenic role for PATZ1 in p53-null cells.

a

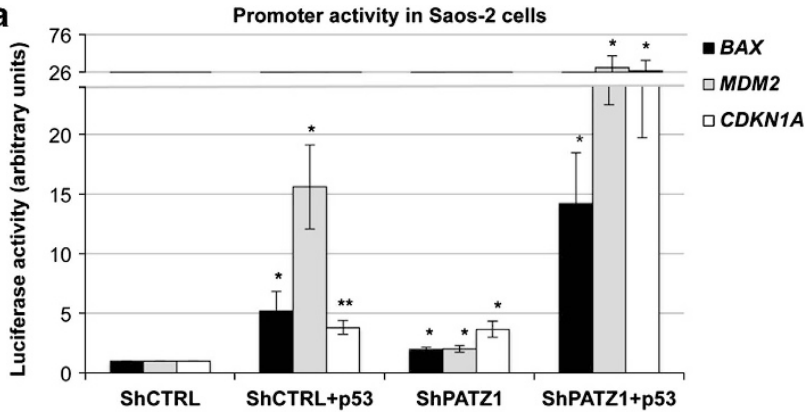

b
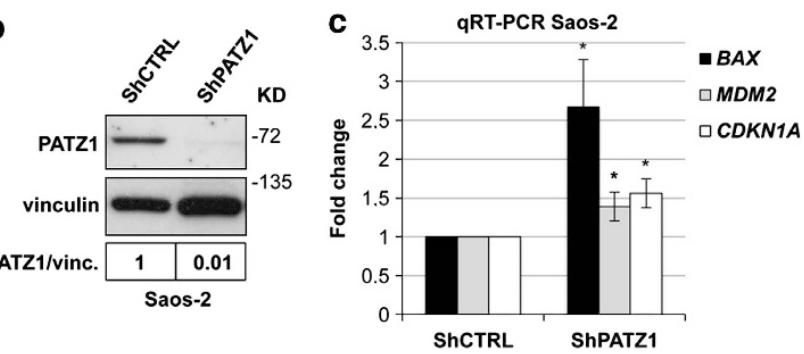

Figure 4 PATZ1 knockdown upregulates $p 53$ activity and p53-target genes in Saos-2 cells. (a) Promoter activity of the BAX, CDKN1A and MDM2 genes as assessed by luciferase assay in Saos-2 cells interfered for PATZ1 (ShPATZ1) and their backbone vector expressing control (ShCTRL). Where indicated, p53 was cotransfected with the reporter plasmids. The data shown express the mean values \pm S.E. of three independent experiments. Asterisks indicate the statistical significance versus promoter basic activity. (b) Western blot analysis of PATZ expression in cells shown in a. Vinculin expression was evaluated as a loading control. Relative expression levels, compared with ShCTRL cells and normalized with respect to vinculin, are indicated on the bottom. (c) qRT-PCR analysis of $B A X$, MDM2 and CDKN1A gene expression in cells shown in $\mathbf{a}$ and $\mathbf{b}$. The data shown express the mean values \pm S.E. of three independent experiments
PATZ1 can act either as a pro-apoptotic or anti-apoptotic factor depending on the cellular context. One of the main functions of p53 is the positive modulation of apoptosis in response to genotoxic conditions. ${ }^{20}$ Therefore, to investigate the biological consequences of the functional interaction between PATZ1 and p53, we analysed the potential role of PATZ1 in apoptosis. To this aim, we analysed PARP and Caspase-3 cleavage in wt and Patz1-ko MEFs using western blot. As shown in Figure 5a, the expression of the cleaved Caspase-3, representing cells that undergo apoptosis, is lower in Patz1 $1^{-1}$ MEFs compared with wt controls. Similarly, Patz1 ${ }^{-1-}$ cells also showed reduced levels of the cleaved PARP protein, another hallmark of apoptosis. Conversely, no significant differences were observed for heterozygous cells compared with wt controls. These results are consistent with reduced spontaneous apoptosis in Patz1null MEFs compared with wt controls. Moreover, the percentage of mortality evaluated by counting viable cells after exposure to 5-fluorouracil (5FU), a known pro-apoptotic chemotherapeutic agent acting in both a p53-dependent and a p53-independent manner, ${ }^{25}$ was significantly reduced, or tended to be reduced, in Patz1 ${ }^{-/-}$and Patz1 ${ }^{+/-}$MEFs, respectively, compared with wt controls (Figure $5 d$ ). These results suggest a pro-apoptotic role for PATZ1 in these cells.

Next, we analysed 5FU-induced apoptosis in HEK293 and Saos-2 cells interfered or not for PATZ1. As shown in Figure 5e, PATZ1 silencing enhanced sensitivity of Saos-2 cells to the pro-apoptotic treatment. This was consistent with the increased levels of the Bax gene in Saos-2 cells interfered for PATZ1. Conversely, no significant differences were observed in HEK293 cells likely because of the high data variability among independent experiments. However, in each experiment we observed a high tendency of HEK293interfered cells to be more sensitive to the chemotherapeutic treatment compared with control cells (Supplementary Figure S5). All together, these results suggest a dual pro-apoptotic/ anti-apoptotic role for PATZ1, which depends on the cellular context, and open new interesting therapeutic possibilities in osteosarcomas.

\section{Discussion}

The development of several malignancies in Patz1-ko mice suggests a key role for the PATZ1 gene in tumorigenesis, ${ }^{13}$ which appears to be confirmed by its frequent misexpression in human cancer. ${ }^{3,16-18}$ We employed an Ab array screening to identify the proteins interacting with PATZ1 in order to unveil the mechanisms by which PATZ1 is involved in tumourigenesis. From this screening we identified the tumour suppressor p53 in the same complex with PATZ1. Subsequently, we have studied the functional consequences of this interaction demonstrating that PATZ1 interference (in HEK293 cells carrying a wt p53) results in the inhibition of the p53 activity on the transcriptional regulation of p53-target genes, including $B A X, M D M 2$ and $C D K N 1 A$, thus suggesting a positive role for PATZ1 on p53 transcriptional activity. Moreover, Patz1-null MEFs show a decreased number of apoptotic cells, either spontaneous or induced by treatment with the 5FU pro-apoptotic drug, compared with wt controls. 
a
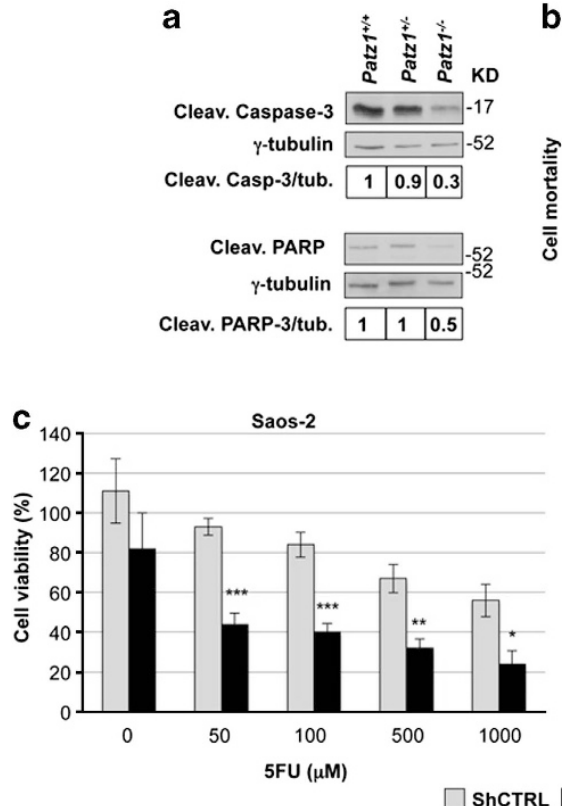
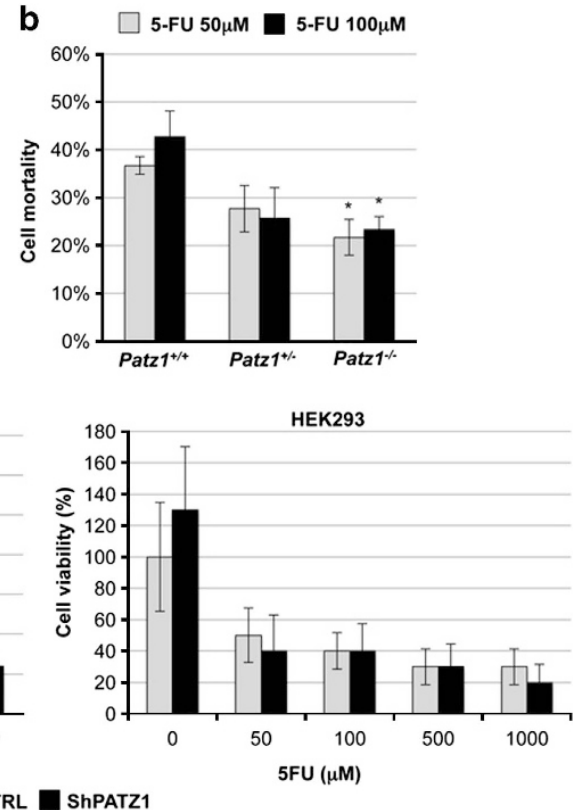

Figure 5 PATZ1 deficiency has a dual anti-apoptotic/pro-apoptotic role depending on the cellular context. (a) Western blot analysis of cleaved caspase-3 and PARP protein expression in Patz $1^{+/+}$, Patz $1^{+/-}$and Patz $1^{-1-}$ MEFs. Relative expression levels, compared with Patz $1^{+/+}$cells and normalized with respect to tubulin, are indicated on the bottom. (b) Cell viability assay in Patz $1^{+1+}$, Patz $1^{+1-}$ and Patz $1^{-1-}$ MEFs treated with different doses of $5 F U$ as indicated on the top. The results show the percentage of cell mortality, expressed as mean values \pm S.E. of three independent experiments, compared with not treated controls. Asterisks indicate the statistical significance compared with Patz1 ${ }^{+/+}$MEFs. (c) Saos-2 and HEK293 cells interfered (ShPATZ1) or not (ShCTRL) for PATZ1 were treated with 5FU at different doses, as indicated at the bottom. The percentage of cell viability is shown as mean \pm S.E. of four independent experiments. Asterisks indicate the statistical significance compared with ShCTRL cells

These results are in agreement with the potential tumour suppressor role of PATZ1, as it enhances the activity of a tumour suppressor gene and its loss confers resistance to apoptosis.

Whereas the positive effect of PATZ1 on p53 activity evidences a clear tumour suppressor function of PATZ1, we also describe an oncogenic potential for PATZ1. Apparently, this occurs when PATZ1 works in absence of p53. Indeed, PATZ1 knockdown upregulates transcriptional activity and expression of the $B A X, M D M 2$ and $C D K N 1 A$ genes in the p53-null Saos-2 cells, and enhances their sensitivity to 5FU pro-apoptotic treatment. Consistently, PATZ1 expression in another p53-null cancer cell line (H1299) causes downregulation of BAX promoter activity. This is in agreement with previous results showing that siRNA downregulation of PATZ1 increases sensitivity of glioma cells (mostly carrying a mutant p53 gene and resistant to conventional chemotherapy) to apoptotic stimuli. ${ }^{19}$ Therefore, we can speculate that targeting of PATZ1 in p53-null tumours, which are mostly resistant to conventional chemotherapic treatment, could be envisaged as an adjuvant therapy to improve the sensitivity of the cancer cells to the conventional chemotherapy. Conversely, if a tumour retains the wt TP53 gene and PATZ1 is underexpressed, increasing the expression of $P A T Z 1$ could enhance p53 activity, thus improving induction of the apoptotic process.

It has been previously shown that HMGA1, a protein interacting with PATZ1, binds to p53 and inhibits its apoptotic activity. ${ }^{21,23}$ It would be interesting to determine whether the interaction between PATZ1 and HMGA1 might interfere with the activity of p53. We can hypothesize that in tumours carrying a wt TP53 gene, the balance between HMGA1 and PATZ1 protein levels might have opposite effects on the activity of p53 and, consequently, in tumourigenesis and in response to anticancer treatments. It is noteworthy that in differentiated thyroid cancer, which shows a very low frequency of mutations in the TP53 gene but a reduced p53 activity, ${ }^{26}$ HMGA1 is overexpressed ${ }^{27}$ and PATZ1 is downregulated compared with normal thyroid tissue (Chiappetta et al., manuscript in preparation).

Recent deep-sequencing analyses confirmed earlier reports of TP53 somatic mutations in $\sim 20 \%$ of diffuse large B-cell lymphomas (DLBCLs), ${ }^{28-30}$ a much lower percentage than in certain non-haematologic malignancies. ${ }^{31,32}$ Nonetheless, $66 \%$ of DLBCLs show decreased abundance of functional p53 and reduced levels of p53 targets. ${ }^{33}$ Therefore, additional bases of $p 53$ deregulation in DLBCLs are still to be defined. Owing to the role of PATZ1 on p53 activity, we could speculate that the downregulation of the PATZ1 gene might be one of the upstream events in the deregulation of p53-dependent pathways in these lymphomas. Consistently, the main malignant phenotype in Patz1-ko mice is the development of DLBCLs, where BCL6, which is known to suppress p53 expression, ${ }^{34}$ is upregulated. ${ }^{13}$

In conclusion, our data demonstrate that PATZ1 is able to interact with p53 and enhance the expression of the genes regulated by $\mathrm{p53}$, then increasing the susceptibility to apoptosis, according to a tumour suppressor role of PATZ. However, the absence of p53 leads PATZ1 to inhibit the same genes, enhancing cell survival. Therefore, our data seem to confirm an oncogenic or anti-oncogenic role for PATZ1 in carcinogenesis depending on the cellular context. 
Materials and Methods

Cell cultures. HEK293, Saos-2 and H1299 cells were grown in DMEM containing 10\% fetal bovine serum (Life Technologies, Monza, Italy), 1\% glutamine (Life Technologies) and 1\% penicillin/streptomycin (Life Technologies). Primary MEFs obtained from 12.5-day-old embryos of timed pregnancies between Patz $1^{+/}$mice, previously described, ${ }^{13}$ were grown in DMEM (Life Technologies) containing 10\% fetal bovine serum (Hyclone, Erembodegem, Belgium), 1\% glutamine, 1\% penicillin/streptomycin and 1\% gentamicin (Life Technologies).

Plasmids, transfections, gene interference and luciferase activity assays. Full-length PATZ1 cDNA (variant 4) for the human PATZ protein was subcloned into the $E c O R I$ site of the pCEFL-HA vector, in frame with the upstream HA tag (pHA-PATZ). Expression plasmid for wt p53 (pCAG-p53) has been described previously. ${ }^{35}$ PATZ1 knockdown in HEK293 and Saos-2 cells was carried out by stable transfection of specific sh-RNA for human PATZ1 (KH08765P; Qiagen, Milano, Italy) after selection in $1 \mu \mathrm{g} / \mu \mathrm{l}$ puromycin. pBAXluc, pMDM2-luc or p21-luc reporter vectors have been described previously. ${ }^{21}$ All transfections were carried out by Lipofectamine 2000 (Life Technologies) according to the manufacturer's protocol. For Luciferase assays, the PCMV-Renilla plasmid (Promega, Mannheim, Germany) was co-transfected with pHA-PATZ and/ or pCAG-p53. Luciferase and Renilla activities were assessed with the Dual-Light Luciferase system (Promega), according to the manufacturer's protocol, $48 \mathrm{~h}$ after the transfection. Luciferase activity was normalized for the Renilla activity. All the experiments were performed at least three times in duplicate or triplicate and the mean \pm S.E. was reported.

Ab array. We used an Ab array filter (Hypromatrix Incorporation, Worcester, MA, USA) in which 100 antibodies, including those against proteins involved in cell cycle regulation, apoptosis and signal transduction pathways, are immobilized on a membrane, at predetermined positions, and retained their capabilities of recognizing and capturing antigens. After incubation with total cell lysates from HEK293 cells overexpressing HA-PATZ, an immunoblot assay was performed following the manufacturer's instructions using an HRP-conjugated anti-HA Ab (sc-805 HRP; Santa Cruz Biotechnology, Dallas, TX, USA).

Protein extraction, IP and western blotting. For protein extraction cells were lysed in lysis buffer containing 1\% NP-40, 1 mM EDTA, $50 \mathrm{mM}$ Tris-HC (pH 7.5) and $150 \mathrm{mM} \mathrm{NaCl}$, supplemented with complete protease inhibitors mixture (Roche, Monza, Italy). Total proteins were separated on a 8-10\% SDS PAGE and transferred to nitrocellulose membranes (GE Healthcare, Milano, Italy) by electroblotting. Membranes were blocked with $1 \times$ TBS, $0.1 \%$ Tween- 20 with $5 \%$ BSA and incubated with antibodies. The antibodies used were as follows: antip53 (DO-1/sc-126 mouse monoclonal, Santa Cruz Biotechnology; and Ab7 sheep polyclonal, Calbiochem, Billerica, MA, USA), anti-PATZ (polyclonal Ab raised against a conserved peptide recognizing all PATZ isoforms of mouse and human origin), anti-HA (sc-805; Santa Cruz Biotechnology), control IgG (sc-2027; Santa Cruz Biotechnology), anti-Myc (sc-40; Santa Cruz Biotechnology), anti-PARP (sc-7150; Santa Cruz Biotechnology) and anti-cleaved Caspase-3 (9664P; Cell Signaling Technology, Danvers, MA, USA). IP and Co-IP procedures were carried out as previously described. ${ }^{36}$

GST pull-down assay. Bacterial expressed GST-p53 mutant GST-p53(13-295) and GST-p53(295-390) proteins were bound to glutathione agarose and used for binding assays with total extracts from HEK293 cells transfected or not with pHA-PATZ expression plasmid. Briefly, proteins in the extracts were allowed to associate with the beads carrying either GST or GST-p53 mutants for $2 \mathrm{~h}$ in NETN buffer (20 mM Tris pH 8.0; $100 \mathrm{mM} \mathrm{NaCl} ; 1 \mathrm{mM}$ EDTA; $0.5 \% \mathrm{NP}-40$ ) at $4{ }^{\circ} \mathrm{C}$. The protein complexes were washed four times in the same buffer, dissociated by boiling in loading buffer, and electrophoresed on a $12 \%$ SDS gel. The proteins were transferred to nitrocellulose and visualized with Red Ponceau staining. Subsequently, they were washed and processed with western blot for PATZ detection as described above.

ChIP and re-ChIP assays. ChIP was carried out with an acetyl-histone H3 immunoprecipitation assay kit (Upstate Biotechnology, Lake Placid, NY, USA) according to the manufacturer's instruction, and subjected to Re-ChIP as previously described ${ }^{36}$ Chromatin samples, derived from Raji or HEK293 cells transfected or not with pHA-PATZ and pCAG-p53, were subjected to IP with the following specific antibodies: anti-PATZ, anti-HA, anti-p53 (Calbiochem). For
qPCR, $3 \mu$ of $150 \mu$ IP DNA was used to amplify BAX, CDKN1A and MDM2 promoter regions. IgG was used as nonspecific controls, and input DNA values were used to normalize the values from quantitative ChIP samples. Percent of IP chromatin was calculated as $2^{\Delta C t} \times 3$, where $\Delta C_{t}$ is the difference between $C_{\text {tinput }}$ and $\mathrm{C}_{\mathrm{tclP}}{ }^{37}$ Primer sequences are available on request.

RNA extraction and quantitative (q)RT-PCR. Total RNA was extracted using TRI-reagent solution (Life Technologies) according to the manufacturer's protocol. qRT-PCR was performed with the SYBR Green PCR Master Mix (Life Technologies) under the following conditions: $10 \mathrm{~min}$ at $95^{\circ} \mathrm{C}$, followed by 40 cycles $\left(15 \mathrm{~s}\right.$ at $95^{\circ} \mathrm{C}$ and $1 \mathrm{~min}$ at $60^{\circ} \mathrm{C}$ ). Each reaction was performed in triplicate in three independent experiments. We used the $2^{-\Delta \Delta C t}$ method to calculate the relative expression levels. ${ }^{38}$ Primer sequences are available upon request.

Cell viability analysis. MEF wt or ko for Patz1, as well as HEK293 and Saos-2 cells, interfered or not for PATZ1 were treated with $5 \mathrm{FU}$ and cell viability was assessed using the CellTiter-Glo Luminescent Cell Viability Assay (Promega), according to the manufacturer's instructions. Percentage of cell mortality was calculated applying the formula 1 - cell survival of treated cells/cell survival of untreated cells.

Statistical analyses. The one-way ANOVA followed by Tukey's multiple comparison test was used to compare groups of experiments. Differences between two sets of data were analysed by two-tailed unpaired $t$-test, where significance levels were set as follows: ${ }^{*} P \leq 0.05$; ${ }^{* *} P \leq 0.01$; ${ }^{* \star} P \leq 0.001$.

\section{Conflict of Interest}

The authors declare no conflict of interest.

Acknowledgements. This work was supported by the Associazione Italiana Ricerca sul Cancro (AIRC-IG5728) and the Ministero Italiano dell'Università e della Ricerca scientifica (MIUR-Prin 2009). TV is recipient of a fellowship from Fondazione Italiana per la Ricerca sul Cancro (FIRC).

1. Fedele M, Benvenuto G, Pero R, Majello B, Battista S, Lembo $F$ et al. A novel member of the BTB/POZ family, PATZ, associates with the RNF4 RING finger protein and acts as a transcriptional repressor. J Biol Chem 2000; 275: 7894-7901.

2. Kobayashi A, Yamagiwa H, Hoshino H, Muto A, Sato K, Morita M et al. A combinatorial code for gene expression generated by transcription factor Bach2 and MAZR (MAZ-related factor) through the BTB/POZ domain. Mol Cell Biol 2000; 20: 1733-1746.

3. Mastrangelo T, Modena P, Tornielli S, Bullrich F, Testi MA, Mezzelani A et al. A novel zinc finger gene is fused to EWS in small round cell tumour. Oncogene 2000; 19: 3799-37804.

4. Costoya JA. Functional analysis of the role of POK transcriptional repressors. Brief Funct Genomic Proteomic 2007; 6: 8-18.

5. Kelly KF, Daniel JM. POZ for effect-POZ-ZF transcription factors in cancer and development. Trends Cell Biol 2006; 16: 578-587.

6. Jeon BN, Choi WI, Yu MY, Yoon AR, Kim MH, Yun CO et al. ZBTB2, a novel master regulator of the $\mathrm{p53}$ pathway. J Biol Chem 2009; 284: 17935-17946.

7. Jeon BN, Kim MK, Choi WI, Koh DI, Hong SY, Kim KS et al. KR-POK interacts with p53 and represses its ability to activate transcription of p21WAF1/CDKN1A. Cancer Res 2012; 72: $1137-1148$.

8. Cho JH, Kim MJ, Kim K, Jand Kim JR. POZ/BTB and AT-hook-containing zinc finger protein 1 (PATZ1) inhibits endothelial cell senescence through a $\mathrm{p} 53$ dependent pathway. Cell Death Differ 2012; 19: 703-712.

9. Morii E, Oboki K, Kataoka TR, Igarashi K, Kitamura Y. Interaction and cooperation of mi transcription factor (MITF) and myc-associated zinc-finger protein-related factor (MAZR) for trascription of mouse mast cell protease 6 gene. J Biol Chem 2002; 277: 8566-8571.

10. Pero R, Lembo F, Palmieri EA, Vitiello C, Fedele M, Fusco A et al. PATZ attenuates the RNF4-mediated enhancement of androgen receptor-dependent transcription. J Biol Chem 2002; 277: 3280-3285.

11. Bilic I, Koesters $C$, Unger B, Sekimata M, Hertweck A, Maschek R et al. Negative regulation of $\mathrm{CD} 8$ expression via $\mathrm{Cd} 8$ enhancer-mediated recruitment of the zinc finger protein MAZR. Nat Immunol 2006; 7: 392-400.

12. Sakaguchi S, Hombauer M, Bilic I, Naoe Y, Schebesta A, Taniuchi I et al. The zinc-finger protein MAZR is part of the transcription factor network that controls the CD4 versus CD8 lineage fate of double-positive thymocytes. Nat Immunol 2010; 11: $442-448$. 
13. Pero R, Palmieri D, Angrisano T, Valentino T, Federico A, Franco $R$ et al. POZ-, AT-hook-, and zinc finger-containing protein (PATZ) interacts with human oncogene $B$ cell lymphoma 6 (BCL6) and is required for its negative autoregulation. J Biol Chem 2012; 287: 18308-18317.

14. Burrow AA, Williams LE, Pierce LC, Wang YH. Over half of breakpoints in gene pairs involved in cancer-specific recurrent translocations are mapped to human chromosomal fragile sites. BMC Genomics 2009; 10: 59 .

15. Valentino T, Palmieri D, Vitiello M, Simeone A, Palma G, Arra C et al. Embryonic defects and growth alteration in mice with homozygous disruption of the Patz1 gene. J Cell Physiol 2012; 228: 646-653.

16. Tian X, Sun D, Zhang Y, Zhao S, Xiong H, Fang J. Zinc finger protein 278 , a potential oncogene in human colorectal cancer. Acta Biochim Biophys Sin (Shanghai) 2008; 40: 289-296.

17. Fedele M, Franco R, Salvatore G, Paronetto MP, Barbagallo F, Pero R et al. PATZ1 gene has a critical role in the spermatogenesis and testicular tumours. $J$ Pathol 2008; 215: 39-47.

18. Yang WL, Ravatn R, Kudoh K, Alabanza L, Chin KV. Interaction of the regulatory subunit of the cAMP-dependent protein kinase with PATZ1 (ZNF278). Biochem Biophys Res Commun 2010; 391: 1318-1323.

19. Tritz R, Mueller BM, Hickey MJ, Lin AH, Gomez GG, Hadwiger $P$ et al. siRNA down-regulation of the PATZ1 gene in human glioma cells increases their sensitivity to apoptotic stimuli. Cancer Ther 2008; 6: 865-876.

20. Zilfou JT, Lowe SW. Tumour suppressive functions of p53. Cold Spring Harb Perspect Biol 2009; 1: a001883.

21. Pierantoni GM, Rinaldo C, Esposito F, Mottolese M, Soddu S, Fusco A. High Mobility Group. A1 (HMGA1) proteins interact with p53 and inhibit its apoptotic activity. Cell Death Differ 2006; 13: 1554-1563.

22. Menendez D, Inga A, Resnick MA. The expanding universe of $p 53$ targets. Nat Rev Cancer 2009; 9: 724-737.

23. Fedele M, Pierantoni GM, Pallante $P$, Fusco A. High mobility group. A-interacting proteins in cancer: focus on chromobox protein homolog 7 , homeodomain interacting protein kinase 2 and PATZ. J Nucl Acids Inv 2012; 3: e1.

24. Jegga AG, Sherwood SP, Carman JW, Pinski AT, Phillips JL, Pestian JP et al. Detection and visualization of compositionally similar cis-regulatory element clusters in orthologous and coordinately controlled genes. Genome Res 2002; 12: 1408-1417.

25. Longley DB, Harkin DP, Johnston PG. 5-fluorouracil: mechanisms of action and clinical strategies. Nat Rev Cancer 2003; 3: 330-338.

26. Malaguarnera R, Vella V, Vigneri R, Frasca F. p53 family proteins in thyroid cancer. Endocr Relat Cancer 2007; 14: 43-60.
27. Chiappetta G, Tallini G, De Biasio MC, Manfioletti G, Martinez-Tello FJ, Pentimalli F et al. Detection of high mobility group. I HMGI(Y) protein in the diagnosis of thyroid tumors: $\mathrm{HMGI}(\mathrm{Y})$ expression represents a potential diagnostic indicator of carcinoma. Cancer Res 1998; 58: 4193-4198.

28. Morin RD, Mendez-Lago M, Mungall AJ, Goya R, Mungall KL, Corbett RD et al. Frequent mutation of histone-modifying genes in non-Hodgkin lymphoma. Nature 2011; 476: 298-303.

29. Pasqualucci L, Trifonov V, Fabbri G, Ma J, Rossi D, Chiarenza A et al. Analysis of the coding genome of diffuse large B-cell lymphoma. Nat Genet 2011; 43: 830-837.

30. Lohr JG, Stojanov P, Lawrence MS, Auclair D, Chapuy B, Sougnez C et al. Discovery and prioritization of somatic mutations in diffuse large B-cell lymphoma (DLBCL) by whole-exome sequencing. Proc Natl Acad Sci USA 2012; 109: 3879-3884.

31. Cancer Genome Atlas Research Network. Comprehensive genomic characterization defines human glioblastoma genes and core pathways. Nature 2008; 455: 1061-1068.

32. Cancer Genome Atlas Research Network. Integrated genomic analyses of ovarian carcinoma. Nature 2011; 474: 609-615.

33. Monti S, Chapuy B, Takeyama K, Rodig SJ, Hao Y, Yeda KT et al. Integrative analysis reveals an outcome-associated and targetable pattern of p53 and cell cycle deregulation in diffuse large B cell lymphoma. Cancer Cell 2012; 22: 359-372.

34. Phan RT, Dalla-Favera R. The BCL6 proto-oncogene suppresses p53 expression in germinal-centre B cells. Nature 2004; 432: 635-639.

35. Baker SJ, Markowitz S, Fearon ER, Willson JK, Vogelstein B. Suppression of human colorectal carcinoma cell growth by wild-type p53. Science 1990; 249: 912-915.

36. Fedele M, Visone R, De Martino I, Troncone G, Palmieri D, Battista S et al. HMGA2 induces pituitary tumorigenesis by enhancing E2F1 activity. Cancer Cell 2006; 9: 459-471.

37. Frank SR, Schroeder M, Fernandez P, Taubert S, Amati B. Binding of C-Myc to chromatin mediates mitogen-induced acetylation of histone $\mathrm{H} 4$ and gene activation. Genes Dev 2001; 15: 2069-2082

38. Livak KJ, Schmittgen TD. Analysis of relative gene expression data using real-time quantitative PCR and the 2(-Delta Delta C(T)) method. Methods 2001; 25: 402-408.

(c) (i) $\odot$ Cell Death and Disease is an open-access journal Cy ${ }_{\mathrm{BY}} \mathrm{ND}$ published by Nature Publishing Group. This work is licensed under a Creative Commons Attribution-NonCommercialNoDerivs 3.0 Unported License. To view a copy of this license, visit http://creativecommons.org/licenses/by-nc-nd/3.0/

\section{Supplementary Information accompanies this paper on Cell Death and Disease website (http://www.nature.com/cddis)}

